\title{
Investigating the effect of detection and
}

\section{classification range and aircraft dynamics on a simplified maritime surveillance scenario}

\author{
$\begin{array}{lll}\text { G. N. } \text { Mercer }^{1} & \text { S. I. Barry } & \text { D. O. Marlow } \\ & \end{array}$ \\ P. Kilby ${ }^{4}$
}

(Received 30 July 2007; revised 17 March 2008)

\begin{abstract}
Airborne maritime surveillance operations are part of Australia's national security. The determination of an efficient route to approach each ship detected to within the classification range is a difficult variation on the classical Travelling Salesman Problem because, for example, the ships are moving. In this article, variations in the detection and classification ranges are investigated, with greater classification ranges resulting in route length reductions of up to $20 \%$. Including a finite turning circle radius for an aircraft can cause in increase in route length of up to $20 \%$ for a turning circle radius of 10 nautical miles.
\end{abstract}

See http://anziamj.austms.org.au/ojs/index.php/ANZIAMJ/article/view/341 for this article, (c) Austral. Mathematical Soc. 2008. Published April 15, 2008. ISSN $1446-8735$ 


\section{Contents}

1 Introduction

C476

2 The maritime surveillance problem

C477

3 Detection and classification ranges

C480

3.1 TSP formulation . . . . . . . . . . . . . . . C480

3.2 Results and discussion . . . . . . . . . . . . . . C481

4 Effect of aircraft dynamics

C483

4.1 Problem formulation . . . . . . . . . . . . . . . C483

4.2 Results and discussion .............. . . C486

5 Conclusion

C489

References

C490

\section{Introduction}

The Australian Defence Force (ADF) and the Australian Customs Service (ACS) conduct surveillance of the Australian Exclusive Economic Zone and the Australian coastline to detect, classify and possibly apprehend vessels engaging in illegal activities, with annual costs to the ACS of $\$ 237$ million [2]. Improving the efficiency of surveillance operations is of obvious economic and national security benefit. Surveillance is undertaken primarily with a variety of aircraft and some ships. The aircraft used include AP-3C Orion (ADF), Dash 8 (ACS), helicopters and small fixed-wing unmanned air vehicles $[2,3]$.

The Defence Science and Technology Organisation (DSTO) sponsored a maritime surveillance search problem at the 2007 Mathematics-in-Industry Study Group (MISG) [7]. The aim was to find a computationally feasible 
search method that maximised ship (target) classifications while minimising the time taken (thus finding the shortest route) to search an Area of Interest (AI). DSTO uses such models to conduct operations research in areas such as tactics development and future capability assessment. The problem was presented and considered as a variation on the traditional travelling salesman problem (TSP).

This article considers a small subset of the maritime surveillance problem and does not claim to solve the entire problem which is beyond the scope of investigation here. Our focus is two-fold: an initial investigation into the impact of variable detection and classification ranges on classification rate and route length; and the effect of including aircraft turning circles on route length. A related article [8] includes a comparison of the efficiency of different search techniques across a range of ship speeds.

Several assumptions are made which will be progressively relaxed in future work in order to provide a more realistic representation of the actual scenario. Examples of issues not considered here are variations in aircraft altitude and target prioritisation (but considered by Grob [4]) and a maximum aircraft flight time (but considered by Marlow et al. [8]). There are also numerous other factors that influence maritime surveillance operations that have not been taken into account such as variable speed aircraft and ships, ships taking evasive actions, changeable weather and sea conditions affecting sensor performance and historical information from earlier flights and shipping records.

\section{The maritime surveillance problem}

An ' $S$ ' shaped search pattern typical of those used in maritime surveillance (for example, in barrier patrols [1]) is shown in Figure 1. The aircraft must visit each waypoint (A, B, C etcetera) but can deviate along the way to investigate detected ships which then need to be classified. Generally ships in 
the AI are detected by sensors onboard the aircraft; predominantly by radar although Electro-Optic and Infra-Red sensors are also used [2]. A typical AI is substantially larger than the detection range (denoted $r_{d}$ ) so the aircraft flies a search pattern to cover as much of the AI as possible. The aircraft approaches the ship to within the classification range (usually visual classification, denoted $r_{v}$ ) and then returns to the predetermined search pattern.

We consider a simplified version of the surveillance scenario where we only investigate the route between two waypoints on the predetermined search path with deviations to visit detected ships. Figure 1 shows a typical route between waypoints $\mathrm{A}$ and $\mathrm{B}$. The problem is complicated since the ships are moving with typical speeds between 0 and 30 knots (nautical miles per hour); aircraft speed is typically between 100 and 300 knots.

The detection range $\left(r_{d}\right)$ depends on the types of sensors used. For simplicity, we assume that if a ship is within radar detection (or classification) range, it is detected (or classified), or else it is not. In reality, radar detection (forming a target track) and classification (determining target type) are not simple tasks and depend on target cross-section, target aspect angle and weather conditions.

As we are considering a subset of the larger problem, namely the effect of the classification and detection ranges, we restrict ourselves to finding the minimum distance travelled whilst classifying all ships that are detected. We do not take into account any budgeting constraints such as fuel usage or aircrew considerations which are considered in other studies $[4,8]$.

A smaller detection range means the predetermined search path is longer to ensure good coverage of the AI, as more sweeps of the region may be needed. The shortest route is also longer since less ships are detected at any given time and therefore there is less information at each time step to determine a shortest route. 

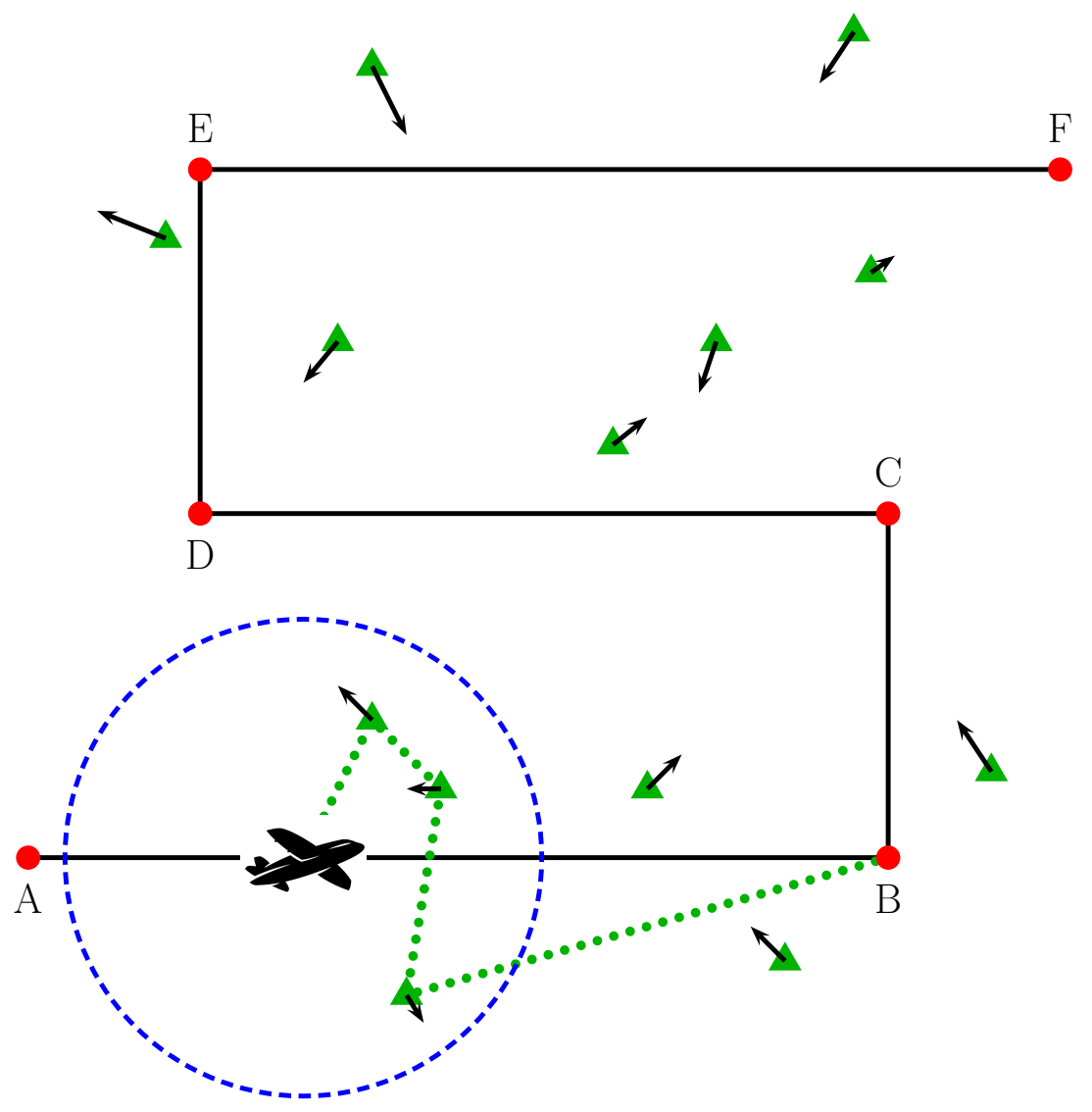

$\hookrightarrow$ ship with velocity vector $\bigcirc$ waypoints

-.-- radar detection zone _.. current optimal route

FiguRE 1: Schematic diagram of the area of interest showing the predetermined base route, the aircraft detection range and an example actual route. 


\section{Detection and classification ranges}

\subsection{TSP formulation}

At any time there may be many ships detected and so the shortest route to visit those ships is sought as a variation on the classical TSP, with new information added as ships are detected and the further complication of moving ships. The shortest route is found by solving a TSP of visiting all detected ships and then flying to the next waypoint. During flight new ships are detected and the shortest route is recalculated to include these.

When ships are detected their velocity (speed and heading) is also determined. If ones assumes the ship velocities are constant, then heuristic algorithms will solve these moving target TSPs $[5,6,9]$ although they are more complicated and slower than stationary TSP heuristics. The assumption of constant velocity is reasonable for large commercial shipping (container ships and the like) but questionable for smaller vessels (fishing boats, smuggling operations). We assume that ship velocity is constant in a short time scale (of the order of minutes) but make no assumptions about their long term velocity. As such we do not use the moving target heuristics mentioned above but rather solve the problem as a sequence of quasi-stationary TSPs at frequent time intervals. At each time step (usually one minute) the current location of the ship is used to determine the TSP tour. We also constrain the aircraft to remain in the AI at all times. Once ships are classified, they are removed from the TsP tour.

The TSP is solved numerically using a Genetic Algorithm (GA) in MATLAB. ${ }^{1}$ Ship location and detection are updated once per minute. The GA uses a population size 10-20 times the total number of ships in the AI. The mutation rate used was 0.85 . The termination condition used was typically

${ }^{1}$ The code is available from the MATLAB Central file exchange, http://www. mathworks.com/matlabcentral. 
around 10 times the number of ships in the AI. An entire simulation, with the aircraft traversing from the beginning waypoint to end waypoint and with recalculations every minute, has a computational run time of less than a second for small number of detects (5-10), rising to around 10 seconds for the largest detection ranges with 25 ships. For low ship density situations with few ships detected at any one time full enumeration of all the possible routes is faster than using the GA and there are also other algorithms that give good solutions in a shorter time frame than the GA used here. Since the simulation's computational time is short in the current work the precise algorithm used is not an issue that needs to be considered here provided the optimal tour is found. In a real scenario the number of ships in the TSP tour is highly variable and can be quite large. Due to this we chose an algorithm that can handle large numbers of ships at the expense of some computational time in the low density situations. Due to space limitations we have concentrated on the results of the method rather than giving a full account of the methods used which can be obtained from the authors.

\subsection{Results and discussion}

Figure 2 is an example of three routes using different detection ranges. These results are for a segment of a surveillance operation typical of an Orion AP$3 \mathrm{C}$ with a straight line distance from A to B of $300 \mathrm{n}$ mile (nautical mile), aircraft speed of 250 knots and 15 ships randomly distributed over the AI. Firstly, $r_{d}$ is set to $500 \mathrm{n}$ mile which gives full knowledge of all ships in the AI and is typical of information from satellite detection when it is possible. Secondly, $r_{d}$ is set to $50 \mathrm{n}$ mile which is typical of radar detection in reasonable weather.

The ship classification range $\left(r_{v}\right)$ is also weather dependent and varies from 0 to $20 \mathrm{n}$ mile. Here we use $r_{v}=3 \mathrm{n}$ mile, meaning the aircraft flies close to the ship, and $r_{v}=20 \mathrm{n}$ mile which is typical of classification in good weather. Table 1 gives the mean length of the calculated route for different 


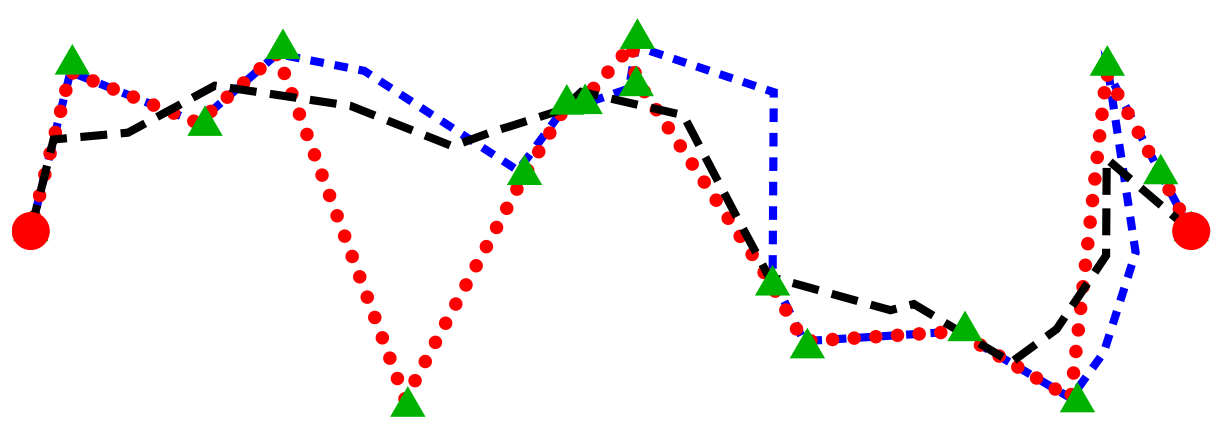

$$
\begin{aligned}
& \text { waypoints } \bullet r_{d}=500, r_{v}=3 \quad-\text { - } r_{d}=50, r_{v}=20 \\
& \triangle \text { ships } \ldots r_{d}=50, r_{v}=3
\end{aligned}
$$

Figure 2: Three optimal routes between two waypoints for 15 ships with various detection and classification ranges: red dotted line $r_{d}=500, r_{v}=3$, blue short dashed $r_{d}=50, r_{v}=3$ and black long dashed $r_{d}=50, r_{v}=20$.

numbers of ships in the AI for both full knowledge $\left(r_{d}=500\right)$ and limited knowledge $\left(r_{d}=50\right)$. The means are over 1000 different random distributions of ships and velocities, with ships travelling at speeds uniformly distributed between 0 and 15 knots and in uniformly random directions. The change in classification range from 3 to $20 \mathrm{n}$ mile gives an approximately $20 \%$ shorter route for the same number of ships classified.

Figure 2 shows that the smaller detection range meant that one outlying ship was not detected. This is a typical outcome unless an effort is made to guarantee complete coverage of the AI. When the aircraft deviates to classify a ship it leaves a gap in the AI that has not been scanned for ships. Table 1 indicates that 10 to $15 \%$ of ships are not detected for the smaller detection range cases. A smaller detection range leads to a longer route as the aircraft must deviate when new ships are detected. When the classification range is larger, then the route is generally smoother and therefore shorter. 
TABLE 1: Optimal route length in n mile with different detection range $\left(r_{d}\right)$ and classification range $\left(r_{v}\right)$ for different numbers of ships in the AI. Also shown are the average number of ships detected and classified. Values are averages of 1000 simulations with standard deviations shown in brackets.

\begin{tabular}{lcccc} 
Number ships & 5 & 10 & 15 & 20 \\
\hline \hline$r_{d}=500, r_{v}=3$ & $391.3(43.0)$ & $533.1(66.3)$ & $683.2(107)$ & $910.3(181)$ \\
& $5(0)$ & $10(0)$ & $15(0)$ & $20(0)$ \\
\hline$r_{d}=50, r_{v}=3$ & $374.8(33.0)$ & $463.8(50.2)$ & $570.8(78.2)$ & $697.6(93.0)$ \\
& $4.50(0.72)$ & $8.84(0.99)$ & $13.5(1.4)$ & $18.6(1.4)$ \\
\hline$r_{d}=500, r_{v}=20$ & $334.5(24.4)$ & $410.4(45.7)$ & $480.7(55.5)$ & $589.0(106)$ \\
& $5(0)$ & $10(0)$ & $15(0)$ & $20(0)$ \\
\hline$r_{d}=50, r_{v}=20$ & $323.7(17.1)$ & $369.5(26.8)$ & $416.9(41.7)$ & $472.6(43.1)$ \\
& $4.50(0.69)$ & $8.95(1.0)$ & $13.4(1.4)$ & $18.2(1.4)$
\end{tabular}

\section{Effect of aircraft dynamics}

\subsection{Problem formulation}

We consider the aircraft heading required to investigate a moving ship with finite turning circle, $r_{c}$, and classification range, $r_{v}$. The aircraft is assumed to have a constant speed $v$ and initial heading $\phi_{i}$, with the ship moving with constant speed $w$, fixed heading $\theta$ and initial position $\left(x_{0}, y_{0}\right)$. The aim is to find the optimal heading of the aircraft $\phi$ and the resultant time to intersection $t_{f}$. We assume the aircraft is at $(0,0)$ when $t=0$ as illustrated in Figure 3. The angles $\xi_{0}$ and $\xi_{1}$ are the parameter angles representing position around the turning circle at the beginning and end of the turn. The angle $\eta$ represents the interception point on the classification circle.

Since the aircraft only has to come within a distance $r_{v}$ of the ship in order to classify it, the aircraft path $(x(t), y(t))$ must intersect the circle around 


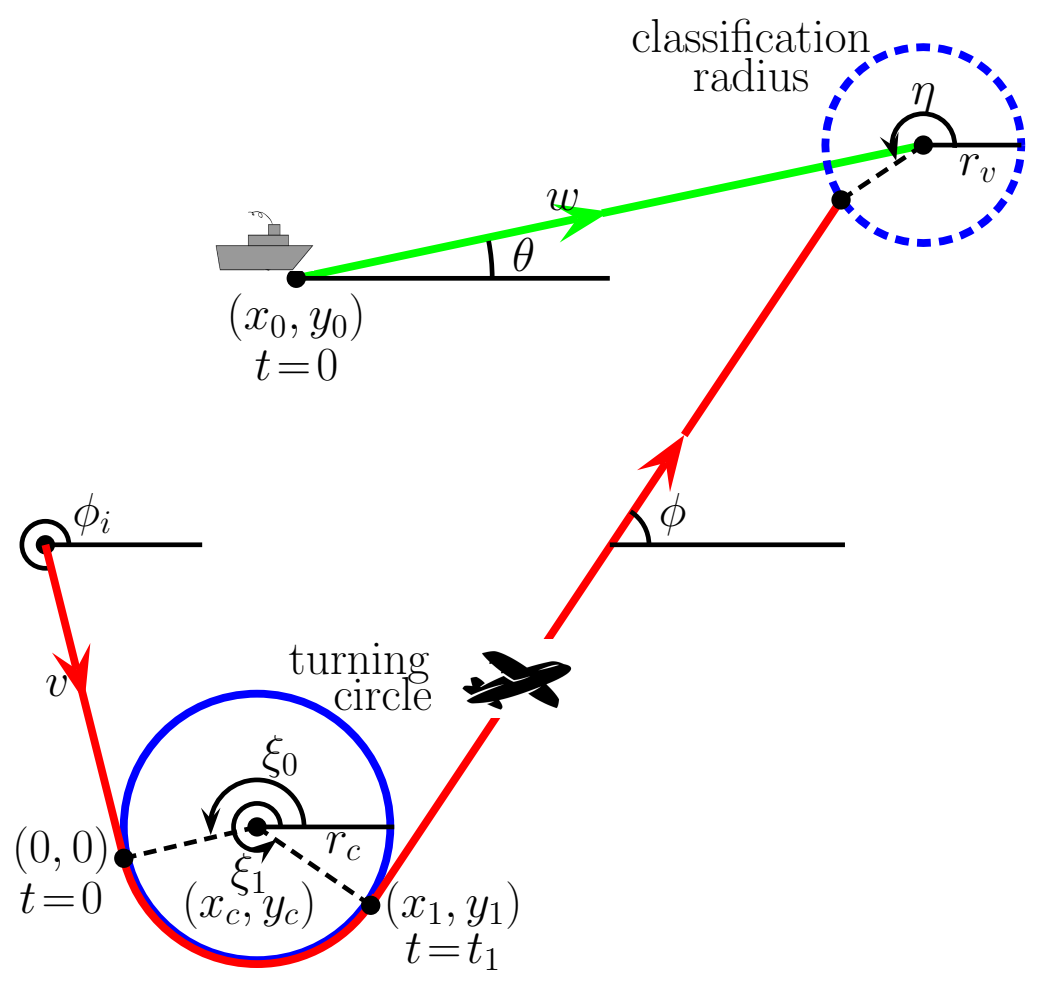

FiguRE 3: An aircraft initially on heading $\phi_{i}$, moving at speed $v$, begins turning at $(0,0)$ at $t=0$ with a turning circle of radius $r_{c}$ with centre $\left(x_{c}, y_{c}\right)$. The turn ends when $t=t_{1}$ at $\left(x_{1}, y_{1}\right)$ and moves along a heading $\phi$ until it reaches its classification range $r_{v}$. The ship moves at speed $w$ on heading $\theta$. 
the ship defined by

$$
\begin{aligned}
& x(t)=x_{0}+w t \cos \theta+r_{v} \cos \eta, \\
& y(t)=y_{0}+w t \sin \theta+r_{v} \sin \eta,
\end{aligned}
$$

where $\eta$ is the angle parameter defining the classification circle (see Figure 3).

The size of the turning circle depends on the aircraft speed and turn rate. The position of the aircraft is governed by three equations:

- for $t \leq 0$, prior to the aircraft beginning to turn,

$$
x=v t \cos \phi_{i}, \quad y=v t \sin \phi_{i} ;
$$

- for $0 \leq t \leq t_{1}$, when the aircraft is turning,

$$
x=x_{c}+r_{c} \cos \left(\frac{v t}{r_{c}}+\xi_{0}\right), \quad y=y_{c}+r_{c} \sin \left(\frac{v t}{r_{c}}+\xi_{0}\right) ;
$$

- for $t>t_{1}$, when the aircraft is on a heading to the ship,

$$
x=x_{1}+v\left(t-t_{1}\right) \cos \phi, \quad y=y_{1}+v\left(t-t_{1}\right) \sin \phi .
$$

By geometry

$$
\xi_{0}=\phi_{i}-\frac{\pi}{2}, \quad \xi_{1}=\frac{3 \pi}{2}+\phi .
$$

The time, $t_{1}$, that the aircraft finishes turning is

$$
t_{1}=\left(2 \pi+\phi-\phi_{i}\right) r_{c} / v
$$

at position $\left(x_{1}, y_{1}\right)$ given by equations (3) with $t=t_{1}$.

Thus equating equations (4) and (1) and doing some simple algebra gives

$$
x_{0}+r_{v} \cos \eta+w t \cos \theta=x_{c}+r_{c} \sin \phi+\left(t-t_{1}\right) v \cos \phi,
$$




$$
y_{0}+r_{v} \sin \eta+w t \sin \theta=x_{c}-r_{c} \cos \phi+\left(t-t_{1}\right) v \sin \phi,
$$

where our unknowns are the aircraft heading $\phi$, the intersection time $t$, and the classification circle angle $\eta$, with $t_{1}(\phi)$ given in equation (6) and $x_{c}, y_{c}, \theta$, $r_{v}, r_{c}, v$ and $w$ all known. This system is solved for $t(\eta)$ and $\phi(\eta)$ by squaring and adding the equations and then $\eta$ is determined by minimising $t(\eta)$.

Hence, we obtain a quadratic in $t: \alpha t^{2}+\beta t+\gamma=0$, with

$$
\begin{aligned}
\alpha & =w^{2}-v^{2} \\
\beta & =2\left(x_{0} r_{v} \cos \eta-x_{c}\right) w \cos \theta+2\left(y_{0} r_{v} \sin \eta-y_{c}\right) w \sin \theta+2 v^{2} t_{1}, \\
\gamma & =\left(x_{0}+r_{v} \cos \eta-x_{c}\right)^{2}+\left(y_{0}+r_{v} \sin \eta-y_{c}\right)^{2}-r_{c}^{2}-t_{1}^{2} v^{2}
\end{aligned}
$$

The resultant solution $t(\phi)$ is substituted into equation (7) to give an implicit equation for $\phi$ which must be solved numerically. Because $\eta$ is still not determined for this most general case, a numerical solution involves iterating over all $\eta \in[0,2 \pi]$ and solving the resultant $\phi$ numerically for each $\eta$ before choosing the value of $\eta$ which minimises $t$. An example using four ships, $r_{v}=5 \mathrm{n}$ mile and $r_{c}=5 \mathrm{n}$ mile is shown in Figure 4.

\subsection{Results and discussion}

The effect of classification range and turning circle on route length is illustrated in Figure 5 for turning circles ranging from 1 to $15 \mathrm{n}$ mile and classification ranges from 0 to $6 \mathrm{n}$ mile. The ships are sufficiently spread such that the classification ranges do not overlap and it is assumed that no new ships are detected and thus added to the tour. This simulation used an aircraft travelling at 240 knots and four ships moving at between 10 and 30 knots spread over an area of roughly $100 \times 100 \mathrm{n}$ mile $^{2}$. As expected, route length increases with turning circle by less than $10 \%$ for $r_{c}=5$, approximately $20 \%$ for $r_{c}=10$ and approximately $25 \%$ for $r_{c}=15$. Similar results occur with different parameters and more ships. Slope changes occur as the best route 


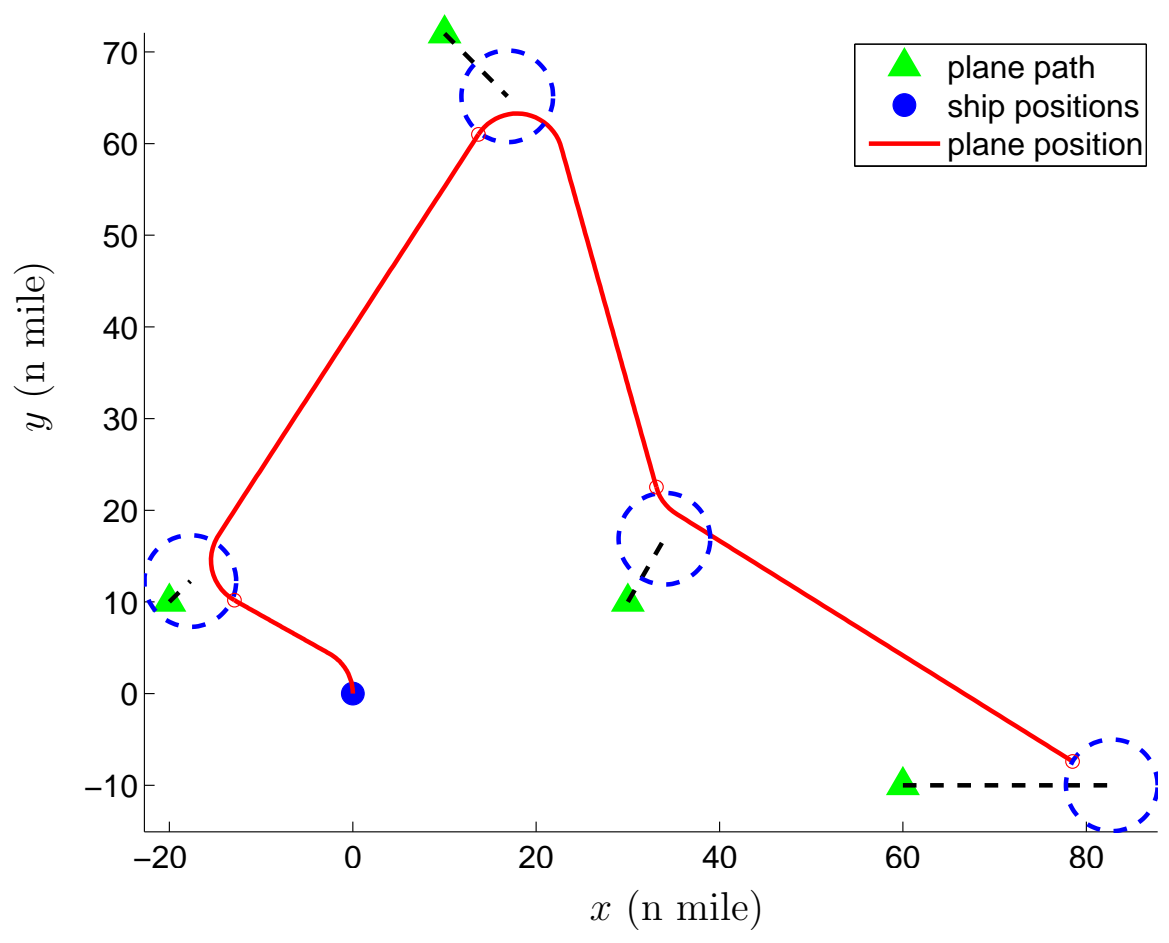

FiguRE 4: Example of an aircraft with a turning circle of radius $5 \mathrm{n}$ mile classifying four ships. 


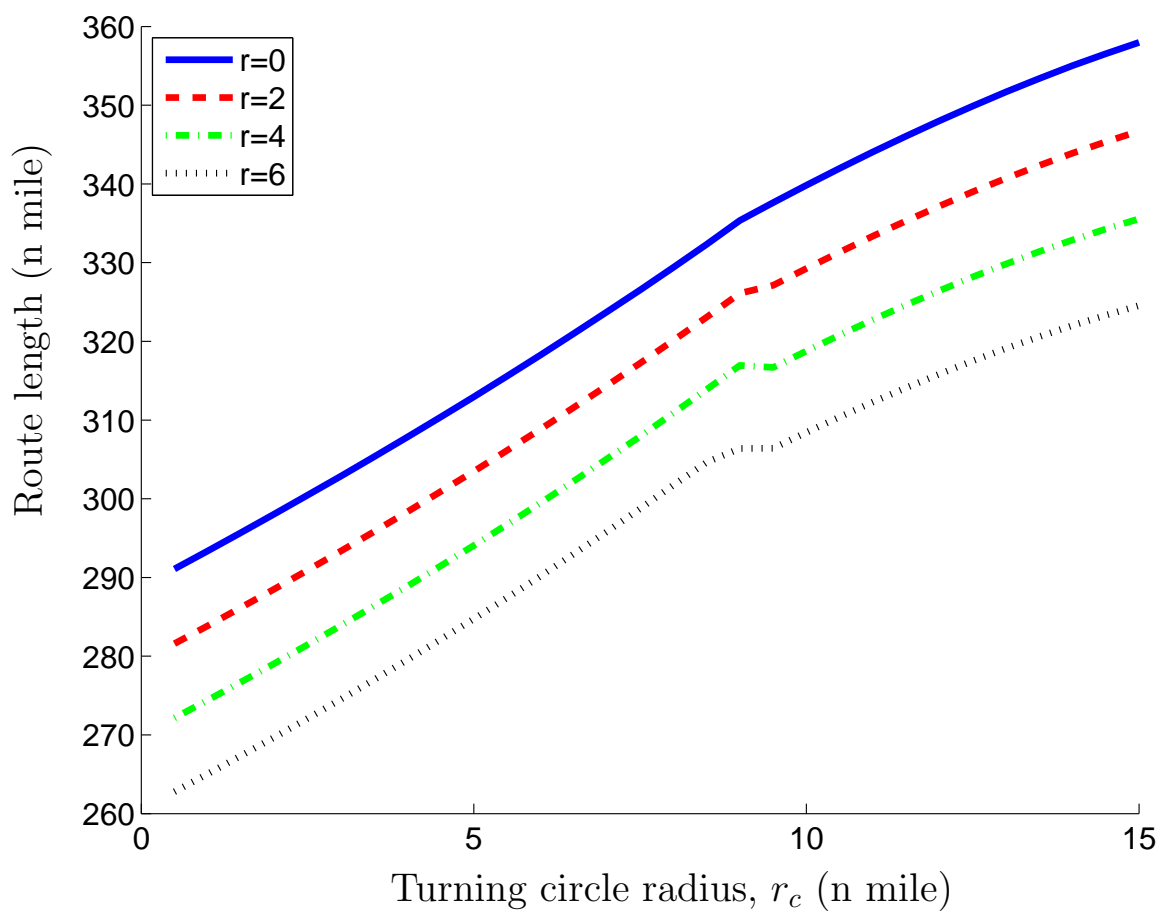

FigURE 5: Effect on distance travelled when increasing turning circle radius for varying classification ranges.

may suddenly involve a different order of ships or an anti-clockwise versus clockwise turn. Notably, the percentage reduction in route length due to classification range variation is independent of aircraft type.

When classifying, an aircraft often needs to approach the ship on critical angles to optimise the capability of on-board sensors. By fixing $\eta$ to such a critical angle (for example, 45 degrees to the ship's path), we numerically showed, for a variety of simulations with similar parameters to that used in Figure 5, that the difference in distance travelled is of the order $1 \%$ for a single ship and strangely a smaller $0.1 \%$ for four ships; errors tend to cancel out rather than accumulate. For some simulations, fixing $\eta$ actually led to a 
decrease in total distance travelled to intercept four ships. This is because our optimisation of $\eta$ to minimise travel length is for an aircraft visiting a single ship; it may not be the optimal value when a second ship is visited and a second turn is required. This non-trivial extension to the problem is the subject of further work.

\section{Conclusion}

We studied a maritime surveillance search scenario using a variation on the traditional travelling salesman technique from operations research. Within this framework we have examined the effect of detection and classification range adopted on the route length needed to classify all detected ships when traversing between two pre-determined waypoints. The difference in classification ranges accounted for a decrease of up to $20 \%$ in total route length for the search aircraft. In the simplified search scenario used in the study approximately $10 \%$ of ships were undetected when the detection range was small, even though classification coverage based on the initially planned route takes in the whole area of interest. Conversely the range of aircraft minimum turning circle radii used in the study accounted for an increase of up to $20 \%$ in total route length for the search aircraft. Clearly both minimum aircraft turning circle radius and classification range can have a significant effect on aircraft route length; aircraft fuel load limits may then impede the search aircraft's ability to classify all ships in the designated area of interest.

Future work will incorporate these outcomes into the travelling salesman solution technique to more correctly determine the shortest route and order of classifying ships. The work described here will be combined with further work to consider coverage of the entire AI. It will also examine the effect that a constraint on the duration of the mission will have on the ability to detect and classify ships. 
Acknowledgements We thank all the participants at the 2007 MISG who worked on this problem, especially Ruth Luscombe, Patrick Tobin and Jason Looker. We also thank Josef Zuk, Simon Goss and Graeme Murray from DSTO for their comments and input and the two reviewers for their thoughtful comments. Joseph Kirk is acknowledged for supplying his Genetic Algorithm implementation for solving the Travelling Salesman Problem to the MatLaB Central file exchange.

\section{References}

[1] Naval Operations Analysis. United States Naval Institute, 1968. C477

[2] Anonymous. Australian Customs Service Annual Report 2005/06. http:

//www . customs .gov . au/webdata/minisites/annualReport0506/. C476, C478

[3] Anonymous. Department of Defence Annual Report 2005/06. http://www.defence.gov.au/budget/05-06/dar/. C476

[4] M. J. H. B. Grob. Routing of platforms in a maritime surface surveillance operation. European Journal of Operational Research, 170:613-628, 2006. doi:10.1016/j.ejor.2004.02.029. C477, C478

[5] C. S. Helvig, G. Robins, and A. Zelikovsky. The moving-target traveling salesman problem. Journal of Algorithms, 43:153-174, 2003. doi:10.1016/S0196-6774(03)00075-0. C480

[6] Q. Jiang, R. Sarker, and H. A. Abbass. Tracking moving targets and the non-stationary travelling salesman problem. In Asia Pacific Symposium on Intelligent and Evolutionary Systems, pages 171-179, 2004. C480 
[7] P. Kilby, P. Tobin, R. Luscombe, S. I. Barry, and R. Hickson. The maritime surveillance problem. In T. R. Marchant, M. Edwards, and G. N. Mercer, editors, Proceedings of the 2007 Mathematics-in-Industry Study Group, pages 32-56, 2008. http://www.uow.edu.au/ informatics/maths/research/misg/UOW025779.html. C476

[8] D. O. Marlow, P. Kilby, and G. N. Mercer. The travelling salesman problem in maritime surveillance-techniques, algorithms and analysis. In L. Oxley and D. Kulasiri, editors, Proceedings of the International Congress on Modelling and Simulation, pages 684-690. Modelling and Simulation Society of Australia and New Zealand, 2007. C477, C478

[9] A. Zhou, L. Kang, and Z. Yan. Solving dynamic tsp with evolutionary approach in real time. In Proceedings of IEEE-CEC2003, volume 2, pages $951-957,2003$. C480 


\section{Author addresses}

1. G. N. Mercer, Applied and Industrial Mathematics, School of Physical, Environmental and Mathematical Sciences, University of New South Wales at the Australian Defence Force Academy, Campbell, ACT 2600, Australia. mailto:g.mercer@adfa.edu . au

2. S. I. Barry, Applied and Industrial Mathematics, School of Physical, Environmental and Mathematical Sciences, University of New South Wales at the Australian Defence Force Academy, Campbell, ACT 2600, Australia.

3. D. O. Marlow, Air Operations Division, Defence Science and Technology Organisation, Fishermans Bend, Victoria 3207, Australia.

4. P. Kilby, National ICT Australia, Northbourne Ave, Braddon, ACT 2601, Australia. 